\title{
Swath Bathymetry Using Phase Difference: Theoretical Analysis of Acoustical Measurement Precision
}

\author{
Xavier Lurton
}

\begin{abstract}
The phase difference principle is widely applied nowadays to sonar systems used for sea floor bathymetry. The apparent angle of a target point is obtained from the phase difference measured between two close receiving arrays. Here we study the influence of the phase difference estimation errors caused by the physical structure of the backscattered signals. It is shown that, under certain current conditions, beyond the commonly considered effects of additive external noise and baseline decorrelation, the processing may be affected by the shifting footprint effect: this is due to the fact that the two interferometer receivers get simultaneous echo contributions coming from slightly shifted seabed parts, which results in a degradation of the signal coherence and, hence, of the phase difference measurement. This geometrical effect is described analytically and checked with numerical simulations, both for square- and sine-shaped signal envelopes. Its relative influence depends on the geometrical configuration and receiver spacing; it may be prevalent in practical cases associated with bathymetric sonars. The cases of square and smooth signal envelopes are both considered. The measurements close to nadir, which are known to be especially difficult with interferometry systems, are addressed in particular.
\end{abstract}

Index Terms-Acoustic scattering, bathymetry precision, interferometry, multibeam echosounder, sea floor bathymetry, sidescan sonar, sonar signal processing.

\section{PRESENTATION}

$\mathbf{M}$ ANY SONAR systems used nowadays for swath bathymetry of the seafloor are based upon the interferometry principle: the elevation angle of a target on the sea floor is measured from the phase difference between the signals received on two separate receivers of the sonar arrays. This processing is more and more commonly used for side-scan sonars, for which it provides an interesting complement to their imaging capabilities at quite a low cost; however, it is also the working principle of many multibeam echosounders. Overviews of the principles, historical development, and applications can be found in classical papers by de Moustier [1], [2] and Denbigh [3].

The basic simplicity of such a measurement principle should not mask the difficulties associated with its practical realization. Phase measurements are quite sensitive ones, leading to largely fluctuating results; the corresponding angle (and bathymetry) evaluation may be quite imprecise. For instance, special care must be taken with problems related to the phase response of

Manuscript received August 18, 1998; revised April 29, 1999, October 4, 1999, and January 24, 2000.

The author is with IFREMER, TMSI/AS, 29280 Plouzané, France.

Publisher Item Identifier S 0364-9059(00)05938-0. the receivers, additive noise, and interference from the sea surface. This type of problem is met with special acuteness for interferometric side-scan sonars and appears to be less penalizing for multibeam echosounders. These issues have been addressed in various previous works [4]-[6], but little has been published about the essential precision bound to the physical constitution of echoes.

In this paper, we propose an analysis of the latter cause of depth measurement inaccuracy. Disregarding the problems related to sea-surface interference [3], to transducer characteristics [4], to phase ambiguities [5], to sound speed profiles variations [6] and to errors bound to ancillary sensors (sonar attitude, sound velocity at the array), we focus here on those problems associated with the physics of the echo formation; in particular, it is shown that, besides the usually considered perturbations brought about by additive noise and decorrelation due to the baseline extent, a noticeable part of the phase fluctuations is due to the structure of the physical signal itself, through a shifting effect of the signal footprint, which is presented and discussed here. The specific problems associated with bathymetry measurements perpendicularly to the seafloor, which are known to be quite penalizing for interferometry systems, are also discussed in this respect.

\section{Phase Difference Bathymetry Measurement}

Measuring the phase difference between two close receivers is a commonly used method in sonar for target localization. The very precisely obtained path length difference then allows one to estimate the target angular direction. Considering the bathymetric sonar configuration of Fig. 1, where $A$ and $B$ are point receivers (with spacing $A B=a$ ) reached by signals emitted from a target point $M$, the phase difference between $A$ and $B$ is

$$
\Delta \varphi_{A B}=k \delta R=k a \sin \gamma=2 \pi \frac{a}{\lambda} \sin \gamma
$$

where $k=(2 \pi / \lambda)$ is the wavenumber, $\lambda$ the wavelength, $\delta R=$ $\overline{M A}-\overline{M B}$ the path length difference, $\psi$ the interferometer tilt angle, and $\gamma=\theta-\psi$ the angle between the target direction and the interferometer axis; in the following, interferometer axis will refer to the median perpendicular to $A B$. The apparent angle $\gamma$ is obtained from measured $\Delta \varphi_{A B}$, finally allowing to determine the position of the point $M$, if the oblique range $R$ is known from the time-of-flight measurement. Practically, it may 


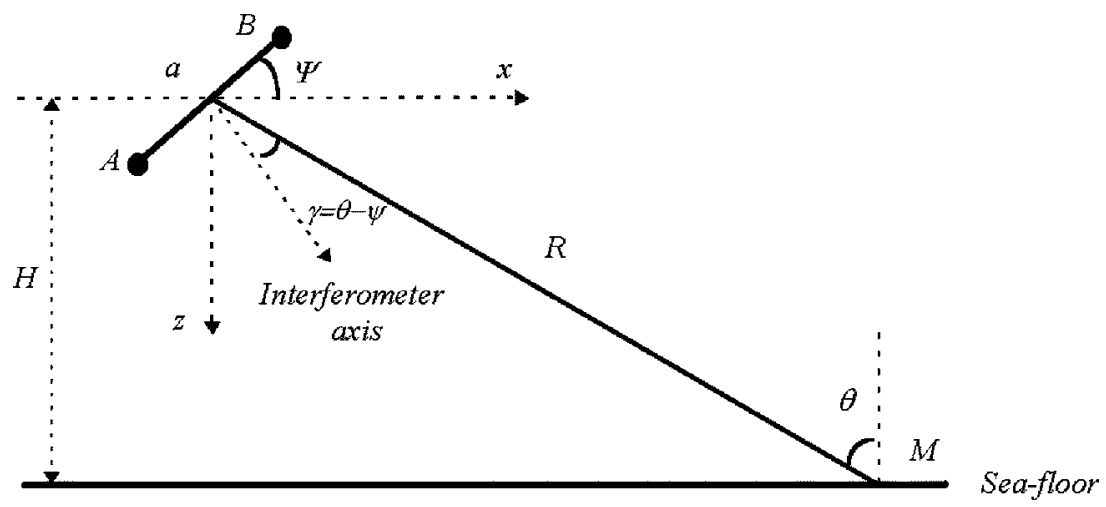

Fig. 1. Geometry of angle and bathymetry measurement using phase difference.

be readily checked that the far-field approximation used in establishing the classical relation (1) is of excellent precision for the case of interferometers with small spacing $a$.

When used in side-scan sonars, the phase difference is usually measured between two identical linear arrays, which sections correspond to points $A$ and $B$ in Fig. 1. The synchronous time series received on both sensors are processed for a phase difference (and hence angle) measurement, with little limitation on the $\gamma$ angle sector (the interferometer is usually baffled whether to select one side or the other of $A B$ ). The same processing is applied in some multibeam echosounders [7]; for a given beam, the interferometry measurement is then performed inside a $\gamma$ sector limited by the beam aperture. The receiving array is split into two "subarrays," each one forming a beam in the main lobe direction; points $A$ and $B$ then correspond to the phase centers of the subarrays. More often, only the null phase difference angle is seeked for in this split-aperture correlator processing [8].

One major problem of the method is that the estimated direction $\theta$ is defined with some ambiguities due to the fact that the phase is measured modulo $2 \pi$. Hence the $\theta$ solution is actually a series of $\theta_{n}$ such as:

$$
\begin{aligned}
\theta_{n}= & \arcsin \left(\frac{\Delta \varphi_{A B}+2 n \pi}{k a}\right)+\psi, \\
& \text { with } n=\cdots-2,-1,0,1,2, \cdots .
\end{aligned}
$$

To prevent this drawback (present as soon as the interferometer spacing is wider than $\lambda / 2$ ), several strategies are possible. In multibeam echosounders [7], the phase difference is measured between signals obtained inside a given formed beam, hence limiting the phase variations to $[-\pi,+\pi]$ and avoiding ambiguities. Another procedure, actually used in many current side-scan systems, is to use two (or more) ambiguous interferometers with different spacings, providing different series of angle solutions, among which only the common values are correct (see Denbigh [3]). A third way of improvement is to measure the phase difference not modulo $2 \pi$, but to "unwind" it, starting from an unambiguous measurement point and following its evolution, imposing continuities at phase jumps (see, e.g., [5]). In the following we shall not consider this issue any more, and shall admit that angle $\theta$ is measured without ambiguities.

\section{ANALYSIS OF MEASUREMENT PRECISION}

\section{A. Angle and Depth Errors}

The angle error in $\theta$ is obtained from a differentiation of (1), replacing $\gamma$ by $\theta-\psi$; it becomes

$$
\delta \theta=\frac{\delta \Delta \varphi_{A B}}{2 \pi} \frac{\lambda}{a} \frac{1}{\cos (\theta-\psi)}
$$

making clear that the angle measurement precision $\delta \theta$ is proportional to the phase difference precision $\delta \Delta \varphi_{A B}$ and is at its best for large values of

- $a / \lambda$, i.e., a wide spacing compared to signal wavelength;

- $\cos (\theta-\psi)$, i.e., a target close to the interferometer axis.

The angle error $\delta \theta$ causes a depth estimation error $\delta z$, given here as a relative value

$$
\frac{\delta z}{z}=\tan \theta \delta \theta=\frac{\delta \Delta \varphi_{A B}}{2 \pi} \frac{\lambda}{a} \frac{\tan \theta}{\cos (\theta-\psi)}
$$

showing that a given angle or phase error is more penalizing at grazing incidences and of less importance near nadir (where $\tan \theta \rightarrow 0$ ).

\section{B. Phase Measurement Precision}

It appears from (3) and (4) that the angle and bathymetry estimation errors (strictly related to the acoustical measurement) are directly proportional to the phase difference measurement error. This point is now examined and analyzed. The basic theory of the target bearing measurement from the phase difference between signals on two subarrays (split-aperture correlator) is developed in Burdic's book [8]. From his equation (13-104), considering an input signal-to-noise ratio (SNR) value $d$, neglecting the individual directivity factors of the subarrays (we are considering here ideal point receivers, in a first step), and replacing his $L / 2$ spacing between the subarray centers by our interferometer spacing $a$, we readily obtain the phase difference standard deviation as

$$
\delta \varphi=\sqrt{\frac{2}{d}}
$$

This classical result (found elsewhere with different approaches, see, e.g., [9]) corresponds to stable signals on the receivers. However, seafloor-backscattered signals are known 
to be quite fluctuating. As a first approximation, the signal amplitudes may be considered as Rayleigh-distributed. This is a correct hypothesis for deep-water low-frequency sonars [10]; on the other hand, we are aware that this is often not justified in the case of high-definition sonars [11], but we assume that the relevance of this classical model is sufficient for our purpose of accounting for the short-term fluctuations (speckle) perturbing phase measurements. Under the Rayleigh-distribution hypothesis, it may be shown (see Appendix I) that the phase standard deviation is increased by a factor $\sqrt{2}$; hence, we shall retain in the following that the phase difference standard deviation is related to the SNR by

$$
\delta \varphi=\frac{2}{\sqrt{d}} .
$$

Note that the SNR $d$ is to be considered at the output of the arrays situated in $A$ and $B$, hence $d=d_{0} G_{D}$ where $G_{D}$ is the array gain for each receiver of the interferometer, and $d_{0}$ is the "physical" SNR at the input of the receivers.

Accounting for the previous geometrical relations, the estimation errors due to noise on measurements of angle and depth are finally

$$
\begin{aligned}
& \delta \theta=\frac{1}{\pi \sqrt{d}} \frac{\lambda}{a} \frac{1}{\cos (\theta-\psi)} \\
& \delta z=x \delta \theta=\frac{x}{\pi \sqrt{d}} \frac{\lambda}{a} \frac{1}{\cos (\theta-\psi)}=\frac{H}{\pi \sqrt{d}} \frac{\lambda}{a} \frac{\tan \theta}{\cos (\theta-\psi)} .
\end{aligned}
$$

The various causes of noise corrupting the signal are considered and analyzed in the following paragraphs.

It appears from (6) that acceptable phase difference fluctuations imply quite high SNR values (for instance, an rms phase difference error $\delta \varphi=10^{\circ}$ corresponds to an SNR of $21 \mathrm{~dB}$ ). However, this may be improved by averaging successive measurement results. This processing may be performed before or after the phase difference computation; for instance, the authors of [12], [13] propose to compute the phase difference from the weighted mean of complex time samples accounting for the SNR level. Moreover, the number of samples used in the filter may be chosen in various ways: the averaging may be performed on a number corresponding either to a constant $\Delta \theta$ (a solution naturally used in multibeam echosounders, allowing one to compensate for the degradation at low grazing angles) or to a constant $\Delta x$ (to keep a constant acrosstrack resolution). The effect of such an averaging will not be considered in the rest of this paper.

\section{Additive Noise}

Additive noise and its various causes (i.e., the sonar itself, its carrier, and the environment) will not be discussed here; only an evaluation of its influence is presented in Fig. 2. Let us consider a $100-\mathrm{kHz}$ sonar, located $50 \mathrm{~m}$ above the seafloor (the input parameters are detailed in the figure caption). Fig. 2 presents the evolution, with lateral range, of the SNR and the corresponding errors in phase, angle, and depth at three realistic levels of ambient noise. It makes clear that the quite high SNR values commonly obtained at vertical and oblique angles $(+20$ to $+100 \mathrm{~dB})$

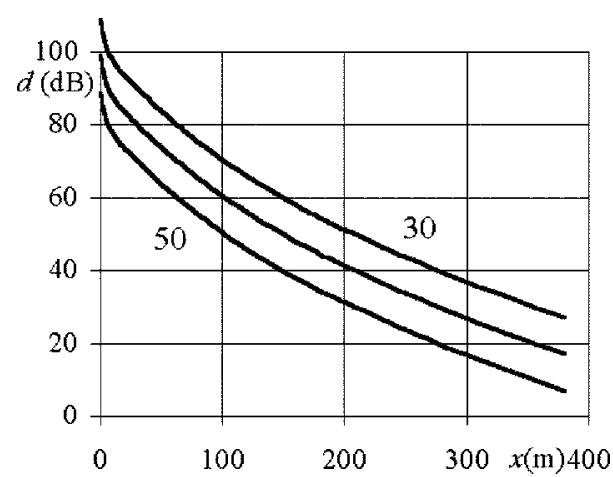

(a)

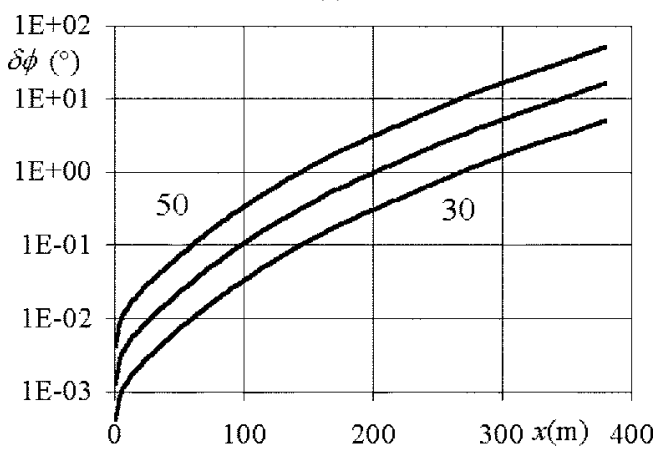

(b)

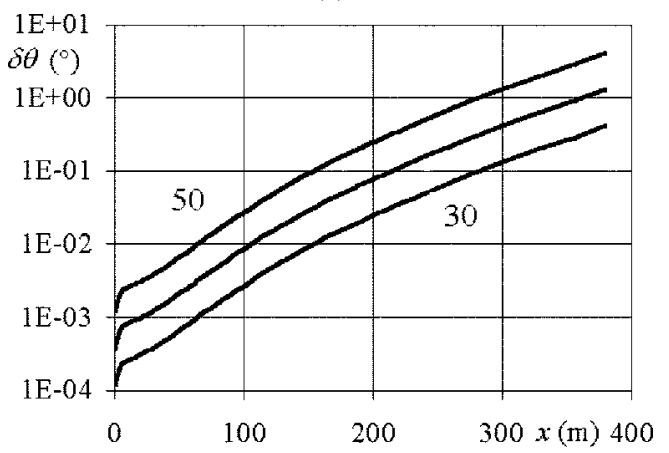

(c)

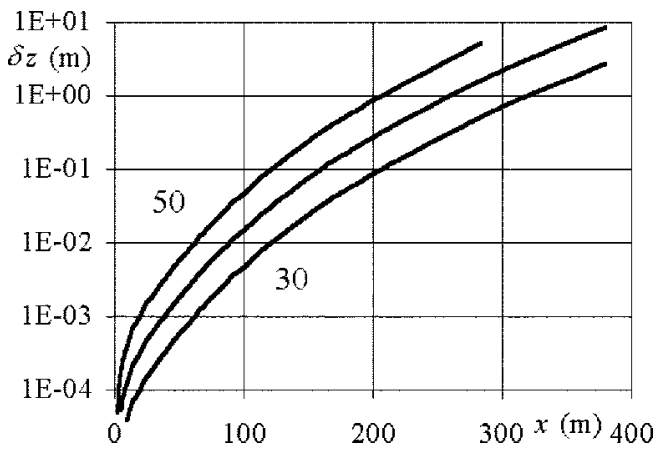

(d)

Fig. 2. Illustrations of the effect of additive noise: (a) computed SNR, (b) phase measurement error, (c) angle measurement error, and (d) altitude error versus range. Input parameters: emitted level $230 \mathrm{~dB} / \mu \mathrm{Pa} / 1 \mathrm{~m} ; f_{0}=100 \mathrm{kHz}$; attenuation $\alpha=30 \mathrm{~dB} / \mathrm{km} ; H=50 \mathrm{~m} ; \theta \in\left[0^{\circ}, 85^{\circ}\right] ; T=0.5 \mathrm{~ms}$; array azimuth aperture $1^{\circ}$; array directivity gain $30 \mathrm{~dB}$; interferometer spacing $a=2 \lambda$ and tilt angle $\Psi=75^{\circ}$; backscattering strength in Lambert's law ref. $-30 \mathrm{~dB} / \mathrm{m}^{2}$. The three curves correspond to noise levels of 30,40 , and 50 $\mathrm{dB} / \mu \mathrm{Pa} / \sqrt{\mathrm{Hz}}$.

lead to very slight phase fluctuations. However, this is in complete disagreement with practical experience. Hence, it has to 
be admitted that other perturbation causes must occur, equivalently to a SNR degradation; such effects are described in the next sections.

\section{Baseline Decorrelation}

All of the above considered the target as a single point scatterer, the dimensions of which have no influence on phase measurement; however, the actual signal footprint extent for bathymetric sonars typically scales from around $10 \mathrm{~cm}$ up to several meters. The echo structure may then be dominated by contributions from individual or clustered scatterers; their summation results in fluctuations in the measured phase difference, and hence in the estimated angle. Even if the average observed direction is given by the signal footprint center, the measured angle variance (related to the scatterer spreading) may be noticeable. Known in radar literature as "glint," this effect in sonar bathymetry was described and discussed in a paper by Jin and Tang [14] as the baseline decorrelation effect. These authors expressed the average cross-correlation $\left\langle s_{A} s_{B}^{*}\right\rangle$ of signals received on $A$ and $B$ as the integral of scatterers contributions along the insonified seafloor segment $\Delta x$; this finally results [14], after normalization, in the following correlation coefficient $\nu$, rewritten here with our notations:

$$
\begin{aligned}
& \nu=\frac{\sin \eta}{\eta} \\
& \eta=\frac{k a}{H} \frac{c T}{4} \cos \theta \cot \theta \cos (\theta-\psi) .
\end{aligned}
$$

This decorrelation process corresponds to an equivalent SNR $d$ given by

$$
d=\frac{\nu}{1-\nu}
$$

The comparisons presented in [14] between the model and experimental results show a good agreement for incident angles between $70^{\circ}$ and $80^{\circ}$. However, the parameters of this configuration are quite particular, since they feature a long emitted signal (with $c T / 2=1.5 \mathrm{~m}$ ) and a low sonar altitude $(5 \mathrm{~m})$ above the seafloor, leading to a value of around 1.6 for $(k a / H)(c T / 4)$ which is the dimensioning factor of $\eta$ in (9). Now considering a high-frequency $(100 \mathrm{kHz})$ bathymetric sonar system such as the one in Section III-C above, with characteristics $T=0.5 \mathrm{~ms}, a=2 \lambda$, and $H=100 \mathrm{~m}$, leads to a much lower value of $(k a / H)(c T / 4) \approx 0.025$. At a given angle, this means an equivalent SNR increase of about 36 $\mathrm{dB}$ compared to the case in [14], since $d \approx\left(6 / \eta^{2}\right)$, which, of course, completely changes the potential influence of the baseline decorrelation effect. Fig. 3 presents the baseline decorrelation equivalent SNR values versus horizontal range, for the system configuration given in Section III-C and two interferometer tilt angles $\psi$. It makes clear that, whatever the angle, the equivalent SNR stays fairly high and is not likely to really affect the phase measurement, except possibly at very short ranges for a tilted interferometer. Therefore, neither this baseline decorrelation effect, considered alone, can be a sufficient explanation for describing the phase fluctuations found in interferometric sonar data.

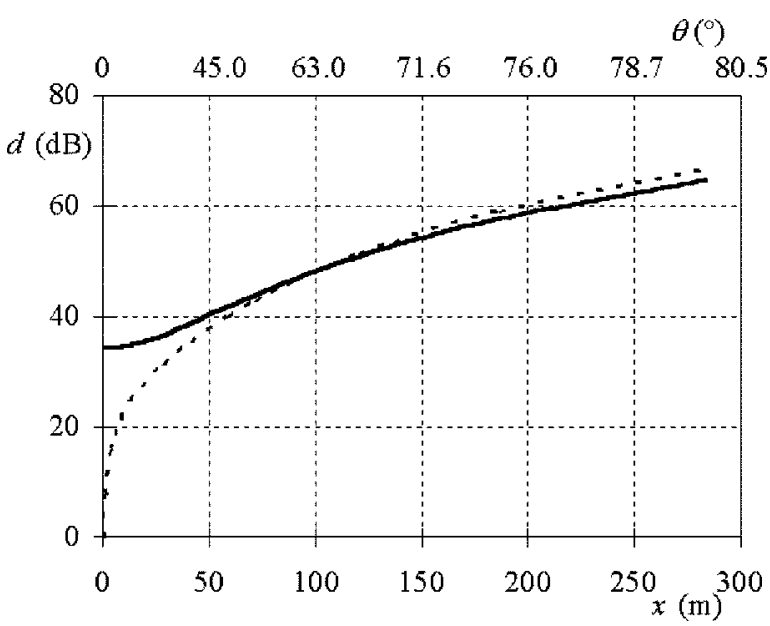

(a)

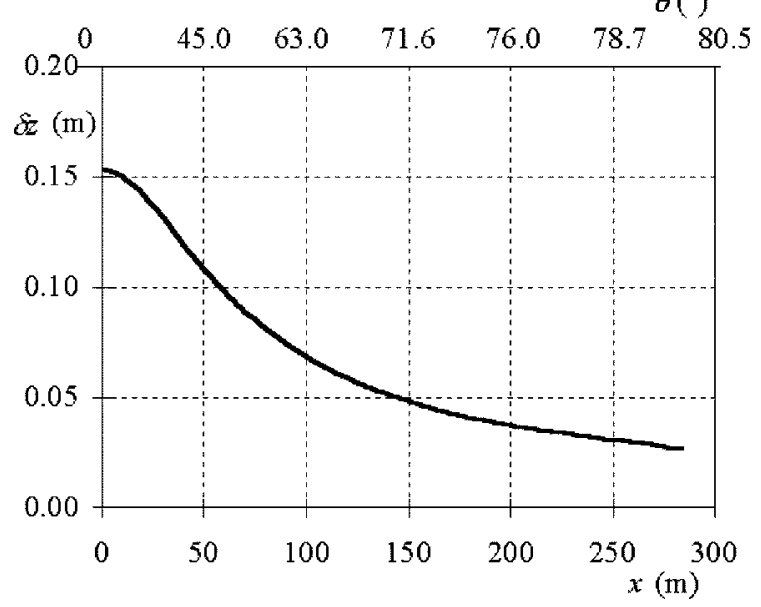

(b)

Fig. 3. (a) Equivalent SNR and (b) depth measurement error caused by the baseline decorrelation effect for a $100-\mathrm{kHz}$ interferometer $a=2 \lambda$ at $H=50$ $\mathrm{m}$ and for two tilt angles of the interferometer: $\psi=90^{\circ}$ (solid line) and $45^{\circ}$ (dotted line). Note that the depth error does not depend on the tilt angle.

\section{SHIFTING FOOTPRINT EFFECT}

\section{A. Introduction}

1) An Optical Analogy: A classical academic experiment in optical interferometry is known as the Young's fringes. It consists in emitting monochromatic light through an opaque shield with two close parallel slots; the radiation from these secondary sources creates on a distant screen periodic interference fringes, the geometry of which is easily computed using a classical development identical to (1) for the phase difference. What is less often emphasized is that the minimum-to-maximum intensity contrast inside the fringes tends to decrease on both sides of the central fringe. This is due to the fact that the light source is not perfectly monochromatic, but features a finite bandwidth, and hence a coherence duration (or equivalent length); therefore, off the interferometer axis, if the path difference from the two slots to the screen gets comparable to this coherence length, the interference phenomenon is degraded [15]. It is thought that a similar phenomenon is affecting phase difference measurements with interferometric sonars.

2) Basic Presentation: In the bathymetry sonar case, the practical phase-difference computation is performed at a 


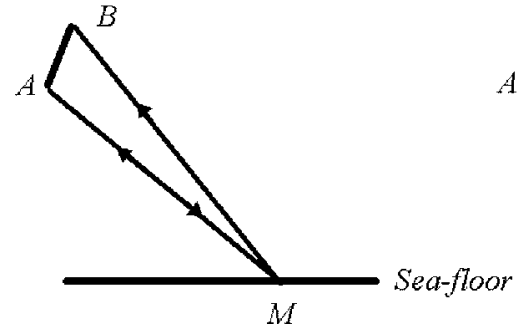

(a)

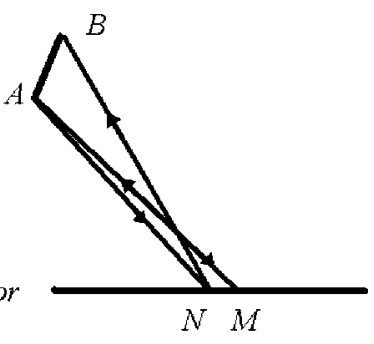

(b)
Fig. 4. (a) Propagation paths associated with a given target point $M$. The emitter being in $A$ and the receivers in $A$ and $B$, the path lengths $A M A$ and $A M B$ are different. (b) Propagation paths associated with a given measurement time $t$ : synchronous echoes on $A$ and $B$ have to come from different target points $M$ and $N$ such as $A M A=A N B$.

given measurement time, between two signals which are the synchronous time series received by $A$ and $B$. This processing requires a sufficient coherence between these two signals. Actually, all of the above developments imply that the signals on the two receivers only differ by their propagation range, hence that their phase difference is a function only of the two path lengths; in other words, at a given time, the two synchronous echoes should come from exactly the same scatterers. This approximation may be unjustified.

Let us consider in Fig. 4(a) the geometry (in the vertical plane) of propagation paths to a given target point $M$ on the seafloor. If $A$ is the emitting point, the echo backscattered by $M$ will obviously reach receivers $A$ and $B$ at different times, since the path lengths $\overline{A M A}$ and $\overline{A M B}$ are not equal (except if $M$ is on the interferometer axis). Put differently, at a given measurement time $t$, synchronous signals received on $A$ and $B$ have to come from two separate target points $M$ and $N$ such as $t=(\overline{A M A} / c)=(\overline{A N B} / \mathrm{c})$; this is represented in Fig. 4(b).

This elementary presentation is for the limit case of an ideally short signal, corresponding locally to a single point scatterer. In the real case of an interferometric sonar, one has to account for the signal duration: the actually insonified area position may slightly differ considering one receiver or the other. This will depend on the receiver spacing, on the altitude, and on the incident angle. The effective signal footprint used in the phase difference measurement is the common part of the two individual footprints; the noncommon parts (supposed uncorrelated) create parasite contributions intervening as noise. We shall first consider a purely geometrical analysis of this, then we shall present some numerical simulation results and discuss the consequences of this phenomenon.

\section{B. Geometrical Description}

The complete configuration is presented in Fig. 5, allowing the geometrical computation of the footprint positions associated to synchronous signals on the two receivers (more detailed developments are given in Appendix II).

The signal footprint length $\Delta x$ is given, at oblique incidences (see Appendix II), by the well-known expression

$$
\Delta x=\frac{c T}{2|\sin \theta|}
$$

where $T$ is the signal duration. Note that $T$ may be either the actual emitted pulse length for classical narrow-band systems or the equivalent duration $T \approx(1 / B)$ ( $B$ being the bandwidth) obtained after pulse compression for sonars using wide-band modulated signals and a correlator in reception.

The footprint shift can be expressed as the variation $\delta x$ of the impact point abscissa due to a position difference $(a \cdot \cos \psi, a \cdot$ $\sin \psi$ ) between receivers $A$ and $B$. It comes readily, in absolute value, as

$$
\delta x=\left|a \cdot \cos \psi-H \tan \theta \pm \sqrt{\left(\frac{H}{\cos \theta}\right)^{2}-(H+a \cdot \sin \psi)^{2}}\right|
$$

where the \pm sign accounts for the sign of $\theta$. Equation (12) is more conveniently written, at oblique incidences, under the approximation

$$
\delta x \approx|a \cdot \cos \psi-a \cdot \sin \psi \cot \theta|=\left|a \frac{\sin (\theta-\psi)}{\sin \theta}\right| .
$$

We consider now that the common part of the signal footprint raising synchronous signals on $A$ and $B$ is effectively used for the phase-difference estimation and is perturbed by the noncommon scatterers, which contribution is perceived as noise. This approach holds if the following hypotheses are made:

- the noncommon scatterer contributions are not correlated with the common ones;

- the common-part scatterers are, on the contrary, perfectly coherent on the two receivers (we neglect, at this stage, the baseline decorrelation);

- the shift is the same at both ends of the insonified segment;

- the ensonified segment lengths are the same for both receivers;

- the ensonification level is constant along the signal footprint (i.e., the signal envelope is square);

- the average target strength of individual scatterers is constant along the $x$ line.

Then the equivalent SNR $d_{s l}$ corresponding to the above shift effect is given by

$$
d_{s l} \approx \frac{\Delta x-\delta x}{\delta x}
$$

where $\Delta x$ and $\delta x$ may be replaced by their respective expressions (11) and (12). Using the approximate form (13), (14) simplifies to

$$
d_{s l} \approx \frac{c T}{2 a|\sin (\theta-\psi)|}-1
$$

This quite simple result may be used for a first discussion. The shift effect is obviously more penalizing (i.e., corresponding to a low equivalent $\mathrm{SNR}$ ) for

- a short signal (hence a small $\Delta x$ ): wide-band signals are unfavorable in this respect;

- directions away from the interferometer axis: a contrario along the axis $(\theta=\psi)$, the equivalent SNR tends to infinity, and the shift effect disappears;

- a large interferometer spacing $a$. 


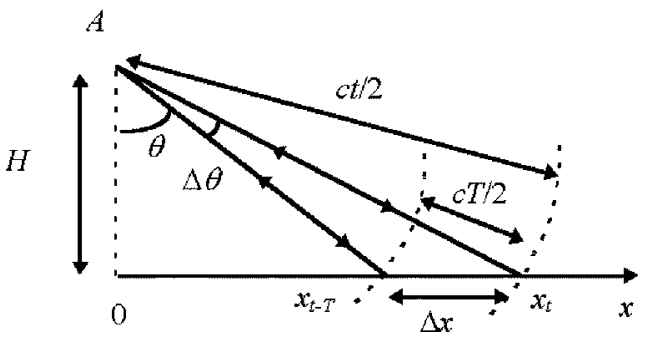

(a)

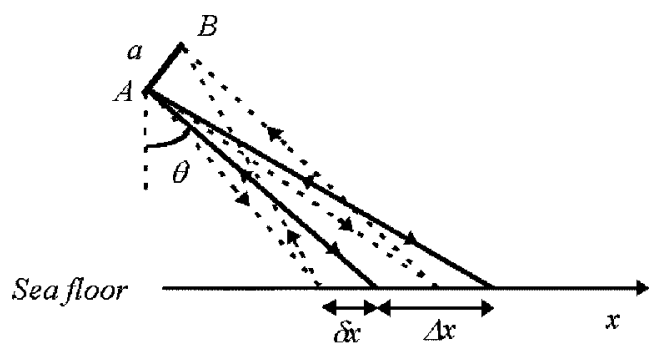

(b)

Fig. 5. Geometry of (a) the signal instantaneous footprint $\Delta x$ and (b) its shift $\delta x$ for separate receivers at a given measurement time.

However, this last point should not be interpreted as an argument in favor of small-spacing interferometers. It is well known [see (3)] that measurement precision is better for wide $A B$ spacing, with an rms error in $1 / a$. However, the shift effect comes and somewhat limits this principle, since its equivalent SNR is proportional to $1 / a$ and, hence, its corresponding measurement error to $\sqrt{a}$. The shifting footprint does not cancel the interest (for accuracy) of using a wide interferometer spacing, but it brings the error down to a variation in $1 / \sqrt{a}$ instead of $1 / a$.

Equation (15) also makes clear that the shifting effect is minimum along the interferometer axis $(\theta=\psi)$. The consequences are:

- for a given interferometer tilt angle, the shift effect will be minimal for one given target angle; inversely, one may imagine tilting the interferometer in order to minimize the shift effect on the whole useful angular sector (see Section IV-E);

- multibeam echosounders with circular arrays [7] avoid the shift effect, since the array portion used for beamforming in a given direction is always physically facing this direction.

\section{Numerical Simulations}

The above shifting footprint model is now checked against numerical simulations. We retained a straightforward concept, based on a classical heuristical model of distributed target points. A series of discrete scatterers is spread on the seafloor line. At a given time (or horizontal range), the active scatterers are determined for each one of the two receivers, using their spherical range. The contributions of the active scatterers are then summed on A and B, and the phase difference between the resultant signals may then be computed. The simulation is run on a significant number (100) of realizations, and the phase standard deviation is finally computed as a function of range. The results are then compared to the rms phase errors computed from (6) with the SNR $d$ expressed according to (14) or (9) and (10).

It is well known that such a heuristic description leads to a Rayleigh's distribution for the resultant signal amplitudes, provided that the signal length is sufficient to include at a given time a large number of scatterers. While this model is well adapted to signals from low-frequency sonars (typically $13 \mathrm{kHz}$ ), it is too rough for systems of higher frequency and resolution, for which amplitude distributions (typically K-laws) are caused by the su- perposition of Rayleigh-like speckle caused by micro-roughness and a Gamma-law modulation by larger scale roughness. We do not try to simulate such phenomena in a first step; it is thought that the speckle effect is the main contributor in the phenomena studied here.

Practically, the scatterer coordinates were taken here to be normally distributed around a basic equidistant grid (here a $2-\mathrm{cm}$ average spacing was used, with a standard deviation of $10 \%$ around this average grid). The amplitudes of the scatterers are normally distributed (standard deviation of $10 \%$ ) while their phases are equally distributed on $[0,2 \pi]$. Since the transverse dimension of the footprint is of little effect in the considered problem of coherence loss, the phenomena are considered in the vertical plane, with no azimuth dependence; this also limits the realism of the simulation, but is sufficient for checking the above concepts of angle spreading and shifting footprint.

The first case presented here is for the same high-frequency system as that described in Section III-C but the sonar altitude is now $50 \mathrm{~m}$. The results in Fig. 6 show a close agreement between the simulated phase standard deviation and the shifting footprint model result, while the baseline decorrelation model predicts very low values. Note the minimum value around 180 $\mathrm{m}$, this range corresponding to pointing along the interferometer axis.

The second simulation case features a different configuration with a longer pulse duration which may be of a lesser concern for actual bathymetry applications, but corresponds to a case of prevalent baseline decorrelation effect. The parameters are kept the same, but the sonar altitude is now $20 \mathrm{~m}$, the signal duration is taken to be $5 \mathrm{~ms}$, and the maximum angle is $85^{\circ}$. The result is given in Fig. 7. The simulated phase difference is seen to follow quite closely the prediction of the baseline decorrelation model, up to a range around $100 \mathrm{~m}$; beyond that, the shifting footprint effect prevails, and the numerical result is in good agreement with the corresponding model. The interest of this particular simulation is to check at the same time that the two effects are accounted for in the simulation algorithm, that different regimes may occur with one or the other error cause prevailing, and that a good agreement is found with both analytical expressions.

\section{Signal Shape Influence}

All of the previous presentation was given for a square envelope signal. This ideal configuration makes the comprehension and the analytical expression of the sliding footprint effect easier, but may lead to its overestimation. Indeed, it is intu- 


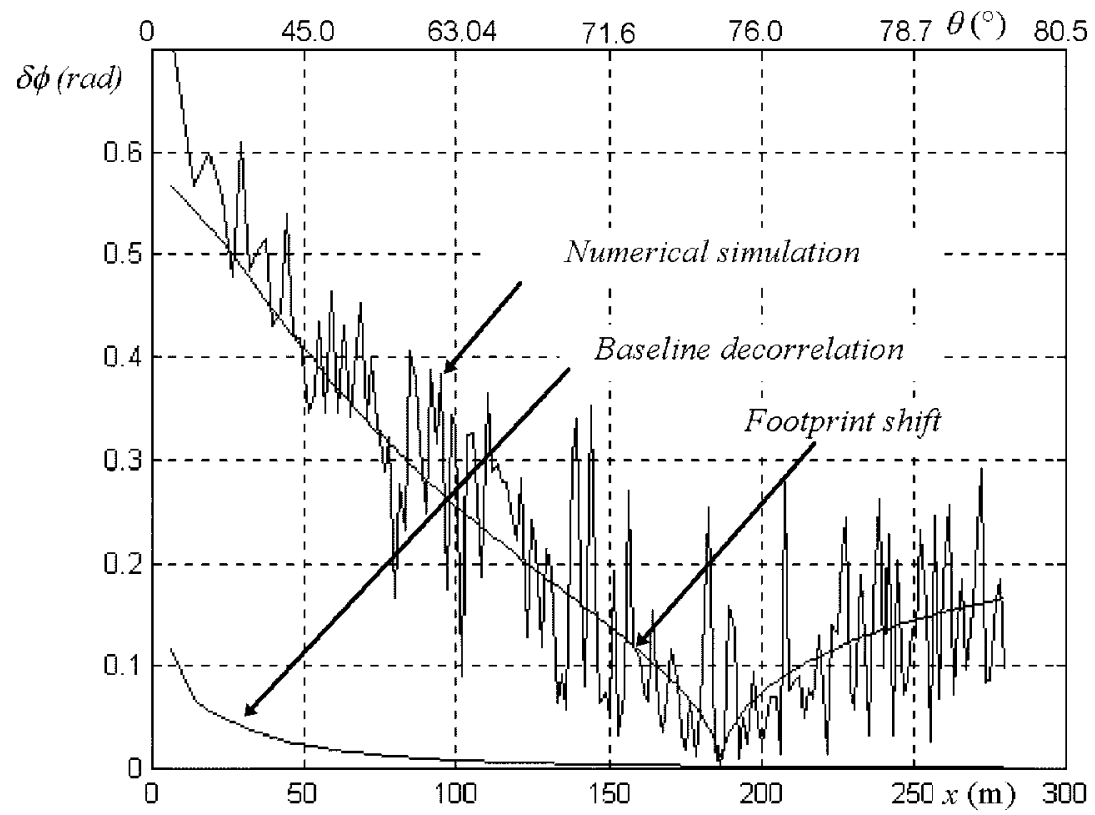

Fig. 6. Standard deviation of simulated phase difference fluctuations for an interferometer, compared to the shifting footprint analytical model, and to the baseline decorrelation effect. The parameters are the same as those in Fig. 2, with $\theta \in\left[0^{\circ}, 80^{\circ}\right]$ and interferometer spacing $a=2 \lambda$.

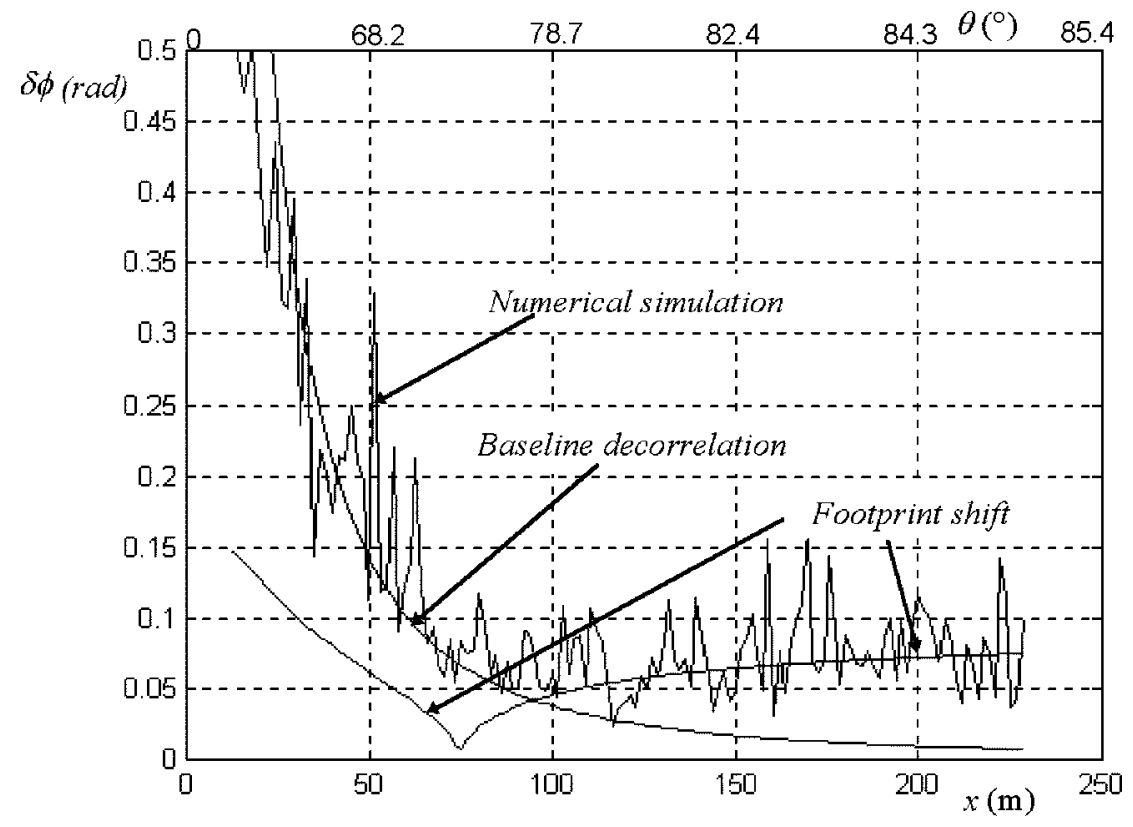

Fig. 7. Standard deviation of simulated phase difference fluctuations for an interferometer, compared to the shifting footprint analytical model, and to the baseline decorrelation effect. The parameters are the same as those in Fig. 6 , but $\theta \in\left[0^{\circ}, 85^{\circ}\right], H=20 \mathrm{~m}$, and $T=5$ ms: the baseline decorrelation here is prevalent for ranges up to $100 \mathrm{~m}$.

itively clear that in this case the "noise" energy associated with the noncommon (transient) parts of the signal is higher than for smooth-shaped envelopes.

Thus, we extend now the above model to arbitrary envelope shapes. While for the square envelope simple geometrical considerations were sufficient, for more complicated envelope shapes, one has to use the complete formulation of the field space correlation [16]. At a given reception time $t$, the cross-correlation coefficient is expressed as

$$
\nu=\left\langle s_{A}^{*} s_{B}\right\rangle / \sqrt{\left\langle s_{A}^{*} s_{A}\right\rangle\left\langle s_{B}^{*} s_{B}\right\rangle}
$$

where $s_{A}$ and $s_{B}$ are the instantaneous signals received at $A$ and $B$. Considering that they are summations of backscattered echoes from scatterers distributed on the $x$ axis, they are expressed as

$$
\begin{aligned}
s_{A} & =\int_{x} S_{A}(x) P(x) e^{j \varphi(x)} d x \\
s_{B} & =\int_{x} S_{B}(x) P(x) e^{j\left(\varphi(x)-\Delta \varphi_{A B}\right)} d x \\
& =e^{-j \Delta \varphi_{A B}} \int_{x} S_{B}(x) P(x) e^{j \varphi(x)} d x
\end{aligned}
$$


where $P(x)$ is the scatterer amplitude distribution along the $x$ axis; similarly to square signals, $S_{A}(x)$ and $S_{B}(x)$ delimit on the $x$ axis the instantaneously active signal footprints for $A$ and $B$, but now accounting for the amplitude modulations induced by the signal envelope shape projected onto the seafloor. $\varphi(x)$ is the phase of the contribution emitted from point $x$ and received at $A$, while $\varphi(x)-\Delta \varphi_{A B}$ is the phase from the same contribution received at $B$. To simplify, we admit here that the phase difference term $\Delta \varphi_{A B}$ is constant over the whole signal footprint width $\Delta x$ (the baseline decorrelation is neglected). The cross-correlation may then be written as

$\left\langle s_{A}^{*} s_{B}\right\rangle=\iint_{x} S_{A}(x) S_{B}\left(x^{\prime}\right) P(x) P\left(x^{\prime}\right) e^{j\left(\varphi\left(x^{\prime}\right)-\varphi(x)\right)} d x d x^{\prime}$.

Considering that the scatterers are uncorrelated and uniformly distributed, the scatterer average cross section $\left\langle P^{2}\right\rangle$ along $x$ may be taken out of the integral, and it becomes

$$
\left\langle s_{A}^{*} s_{B}\right\rangle=\left\langle P^{2}\right\rangle \int_{x}\left\langle S_{A}(x) S_{B}(x)\right\rangle d x .
$$

The $x$ variations of the envelopes $S_{A}(x)$ and $S_{B}(x)$ actually reproduce the time envelope of the signal projected onto the seafloor, with a time delay between them corresponding to the signal footprint shift. Note again that this time envelope may equivalently be the actual emitted envelope of a narrow-band signal, or the envelope obtained after pulse compression of a modulated signal. Finally, introducing the time signal envelope $S(t)$ and expressing (19) in the time domain gives

$$
\left\langle s_{A}^{*} s_{B}\right\rangle=\left\langle P^{2}\right\rangle \int_{\tau}^{T} S(t) S(t-\tau) d t
$$

for a delay $\tau$ corresponding to the shift $\delta x$ and given by

$$
\tau=\frac{T \delta x}{\Delta x} .
$$

The normalized correlation coefficient between $s_{A}$ and $s_{B}$ is finally the autocorrelation function of the signal envelope $S(t)$ for the delay $\tau$ corresponding to the observation time

$$
\nu=\frac{\int_{\tau}^{T} S(t) S(t-\tau) d t}{\int_{0}^{T} S^{2}(t) d t}
$$

and the equivalent SNR is given by (10).

In the case of a square envelope, with $S(t)$ constant for $t \in$ $[0, T]$, one finds easily that (with the time or space formulations)

$$
d_{s l}=\frac{T}{\tau}-1=\frac{\Delta x}{\delta x}-1
$$

i.e., the result (14) of the above geometrical approach. However, besides this limit case, it is evident now that each particular signal envelope shape needs its own development.
As an example, the case of a sine arch envelope $(S(t)=$ $\sin (\pi t / T)$, with $t \in[0, T])$ may be computed analytically with little complexity. The correlation coefficient is

$$
\nu(\tau)=\frac{\int_{\tau}^{T} \sin \left(\frac{\pi}{T} t\right) \sin \left(\frac{\pi}{T}(t-\tau)\right) d t}{\int_{0}^{T} \sin ^{2}\left(\frac{\pi}{T} t\right) d t} .
$$

While the denominator is $T / 2$, the upper integral is developed into the following expression:

$$
\begin{aligned}
\int_{\tau}^{T} & \sin \left(\frac{\pi t}{T}\right) \sin \left(\frac{\pi}{T}(t-\tau)\right) d t \\
= & \frac{(T-\tau)}{2} \cos \left(\frac{\pi \tau}{T}\right)+\frac{T}{2 \pi} \cos ^{2}\left(\frac{\pi \tau}{T}\right) \sin \left(\frac{\pi \tau}{T}\right) \\
& -\frac{T}{2 \pi} \sin ^{3}\left(\frac{\pi \tau}{T}\right) \\
\approx & \frac{T}{2}\left(1-\frac{\pi^{2} \tau^{2}}{2 T^{2}}\right)
\end{aligned}
$$

under the approximation that $\tau$ stays small compared to $T$. The correlation coefficient is then

$$
\nu=1-\frac{\pi^{2} \tau^{2}}{2 T^{2}}
$$

and the equivalent SNR expression comes as

$$
d_{s l}=\frac{2 T^{2}}{\pi^{2} \tau^{2}} .
$$

In order to have a geometrical expression analogous to (15), (27) may be written as

$$
d_{s l}=\frac{2}{\pi^{2}}\left(\frac{c T}{2 a \cdot \sin (\theta-\psi)}\right)^{2} .
$$

The most striking difference between (15) and (28) is the angle (or, equivalently, horizontal range) dependence: the $d_{s l}$ degradation off the interferometer axis is faster for a square envelope (in $\sin ^{-1}(\theta-\psi)$ ) than for a sine $\operatorname{arch}\left(\right.$ in $\sin ^{-2}(\theta-\psi)$ ).

Fig. 8 presents the result of numerical simulations for this signal shape (all the other parameters are the same as those given in Fig. 6), compared with the analytical result (28). The latter is found to provide a very good approximation. Fig. 9 presents the result corresponding to Fig. 7 (prevalent baseline decorrelation regime) with a sine envelope signal. In both cases, the analytical formula for baseline decorrelation was used here with a signal duration equal to $T / 2$, corresponding to the signal envelope effective length. It is seen that the prevalence of the baseline decorrelation effect has been enhanced by the envelope shape, compared to the result of Fig. 8.

Several conclusions are to be drawn from this comparison:

- smoothing the envelope shape noticeably decreases the footprint shift influence, compared to the square envelope case, as it was intuitively perceived;

- the proposed model of the shifting footprint may be readily adapted to this situation, provided that a simple expression of the signal envelope autocorrelation function is available; 


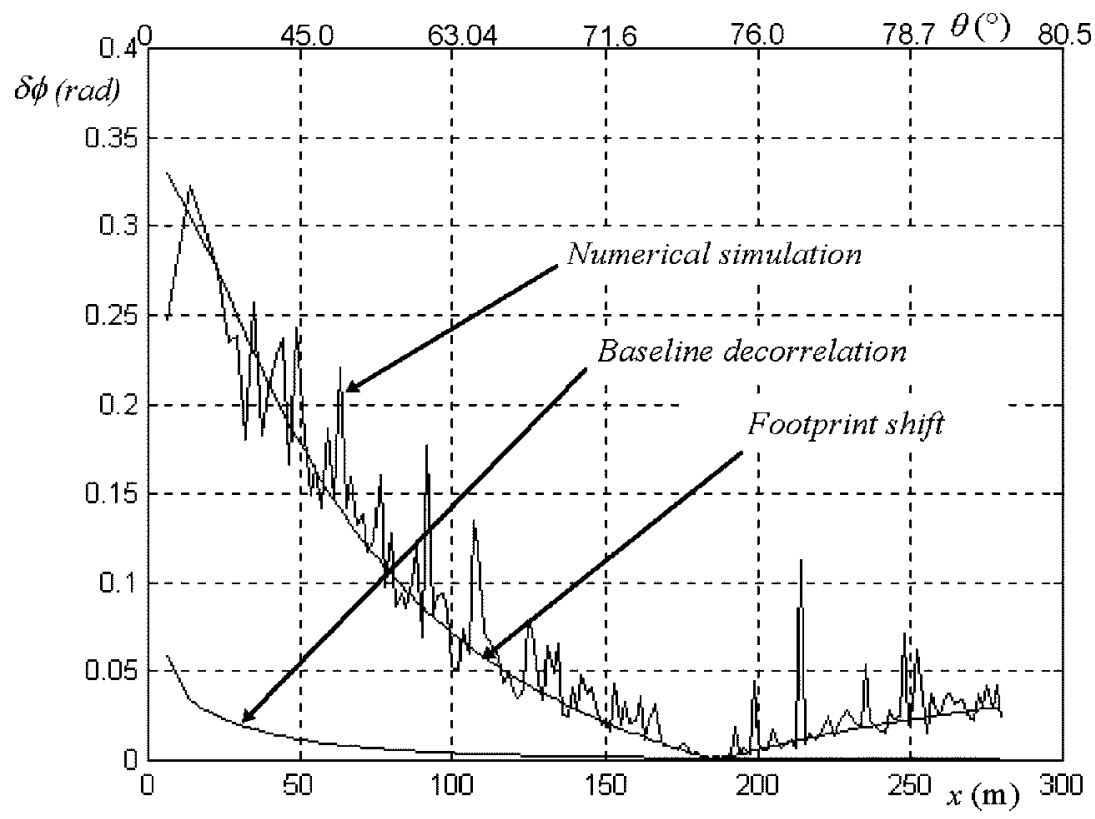

Fig. 8. Standard deviation of simulated phase difference fluctuations for an interferometer, compared to the shifting footprint analytical model, and to the baseline decorrelation effect. The parameters are the same as those given in Fig. 6, but the signal envelope is a sine arch. The baseline decorrelation model uses the effective signal duration.

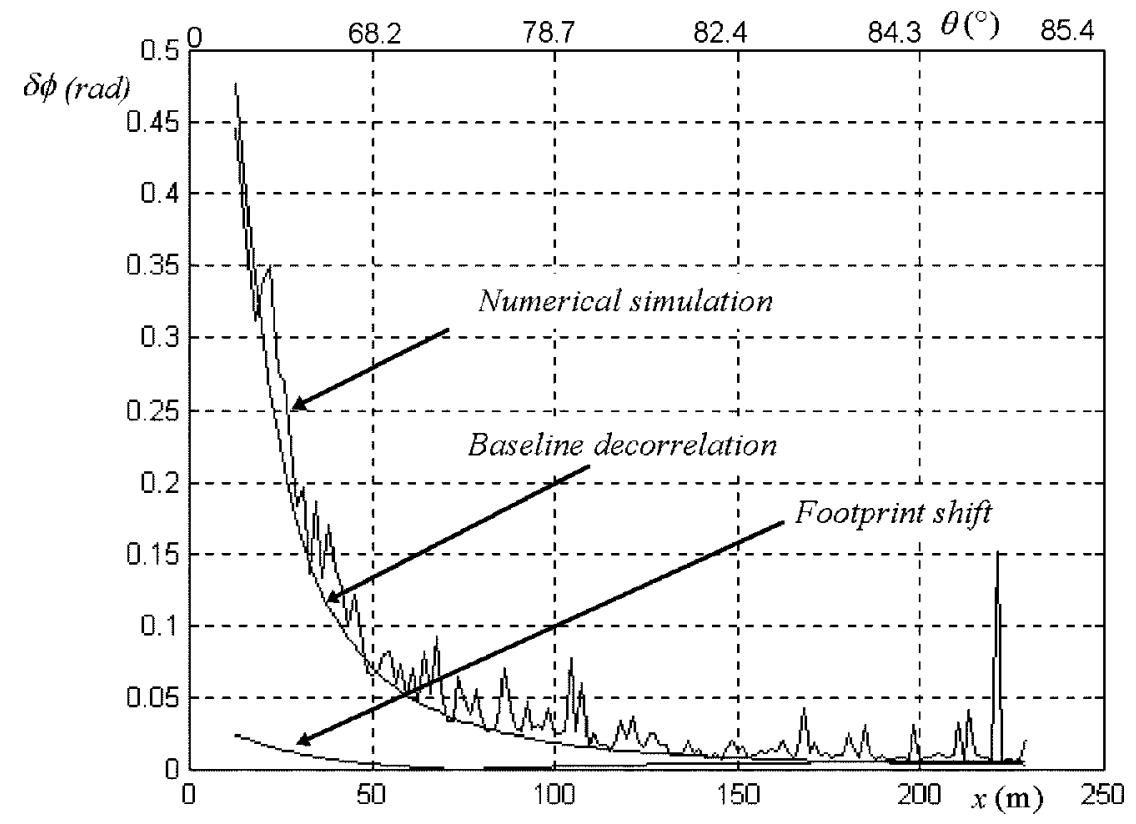

Fig. 9. Standard deviation of simulated phase difference fluctuations for an interferometer, compared to the shifting footprint analytical model, and to the baseline decorrelation effect. The parameters are the same as those given in Fig. 7, but the signal envelope is a sine arch. The baseline decorrelation model uses the effective signal duration.

- the range dependence of the effect is significantly different from the square envelope case according to the envelope shape type.

\section{E. Interferometer Tilt Optimization}

The equivalent SNR due to the shift effect depends on the pointing angle $(\theta-\psi)$. This same angle is featured in (4) giving the bathymetry error from the phase fluctuation. It is then interesting to see what should be the interferometer tilt angle $\psi$ min- imizing such a bathymetry error integrated all along the swath width:

$$
\int_{x \min }^{x \max } \delta z^{2} d x=\int_{\theta \min }^{\theta \max } \frac{\delta z^{2}}{\cos ^{2} \theta} d \theta .
$$

Using expressions of $\delta z$ in (8) and $d_{s l}$ in (15) or (28), the problem is then to minimize, according to $\psi$ the integral:

$$
A(\psi)=\int_{\theta \min }^{\theta \max } \frac{\sin ^{2} \theta|\sin (\theta-\psi)|}{\cos ^{4} \theta \cos ^{2}(\theta-\psi)} d \theta
$$




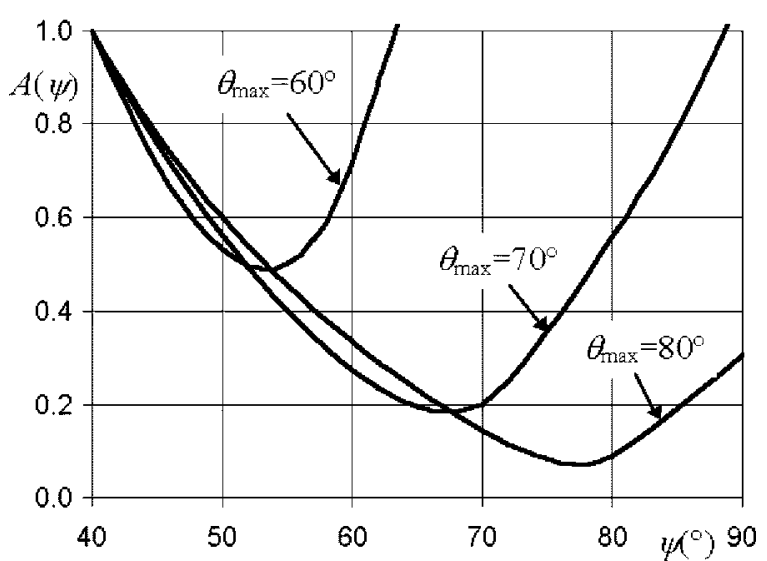

(a)

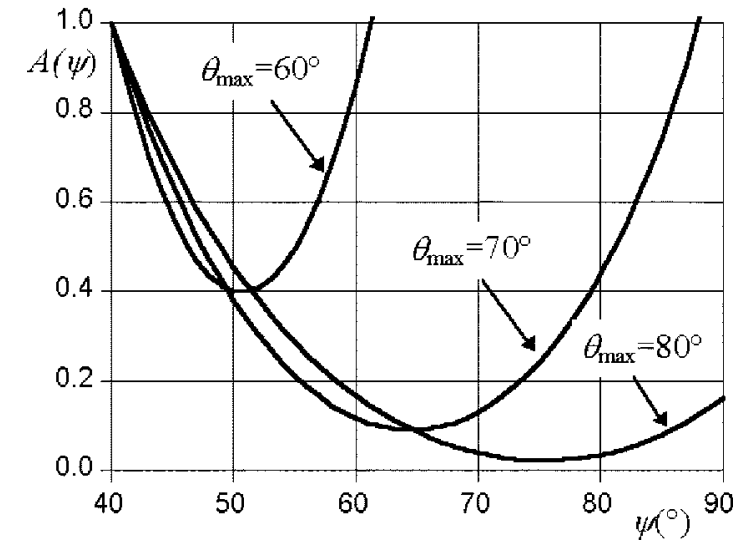

(b)

Fig. 10. Influence of the interferometer tilt angle (abscissa) on the average squared depth error (32), for various values of the extreme angle $\theta_{\text {max }}$. Signal envelopes are (a) square or (b) sine arch. Ordinate scales are arbitrary.

for a square envelope, and

$$
A(\psi)=\int_{\theta \min }^{\theta \max } \frac{\sin ^{2} \theta \sin ^{2}(\theta-\psi)}{\cos ^{4} \theta \cos ^{2}(\theta-\psi)} d \theta
$$

for a sine one. The solution obviously depends on the considered sector $\left[\theta_{\min }, \theta_{\max }\right]$. A numerical evaluation of this integral on $\left[0^{\circ}, \theta_{\max }\right]$ gives an optimal tilt angle slightly below $\theta_{\max }$ (Fig. 10). This is intuitively satisfactory since the interferometer axis is then tilted in order to cancel the shift effect in the direction where the depth errors diverge the most severely. Subsequently, the ideal tilt angle for an interferometer should correspond to the direction of the maximum expected range, say $60^{\circ}$ to $80^{\circ}$. One has to be careful that, even if maximum ranges should correspond to very shallow grazing angles, there is no interest in setting the interferometer completely vertical, since this could degrade the depth measurement near nadir, but also because at long ranges the additive noise effect may prevail upon the shift effect, and minimizing the latter may then not be so crucial.

\section{F. Resulting SNR}

The above equivalent SNR $d_{s l}$ is to be added to the external noise SNR $\left(d_{e x t}\right)$ and to the baseline decorrelation effect (char-

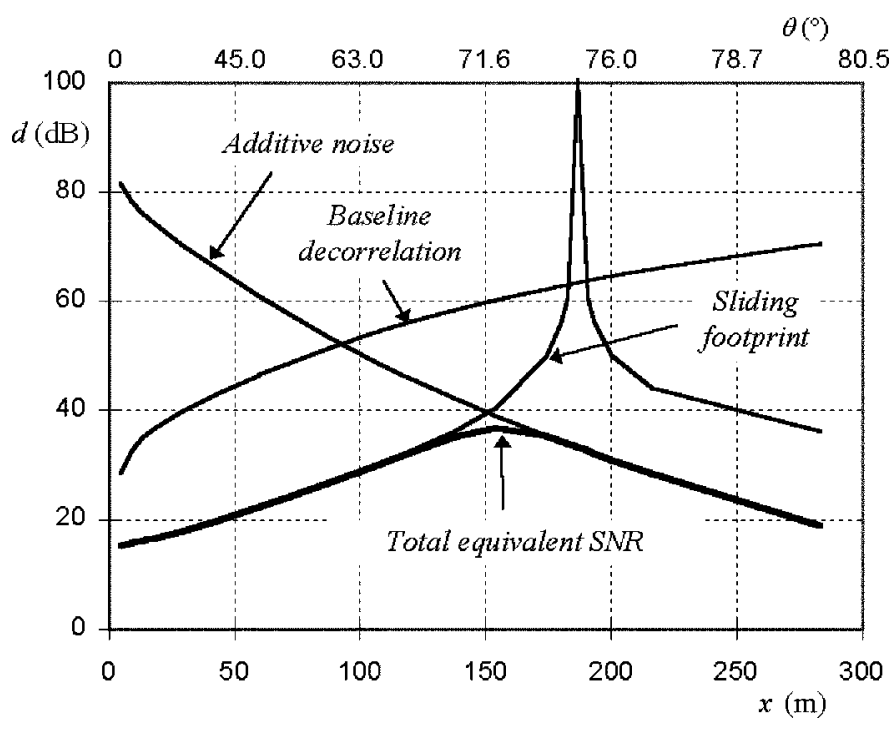

Fig. 11. Resultant SNR for the various effects presented above, with the parameters of Fig. 8. The shifting footprint effect is prevalent up to $150 \mathrm{~m}$; beyond this range, the external noise (here $50 \mathrm{~dB} / \mu \mathrm{Pa} / \sqrt{\mathrm{Hz}}$ ) imposes the SNR. In this configuration, the baseline decorrelation effect remains negligible at all ranges.

acterized by $d_{b d}$ ) to obtain the resulting equivalent SNR, supposing statistical independence between the three phenomena:

$$
d=\frac{1}{\frac{1}{d_{\text {ext }}}+\frac{1}{d_{s l}}+\frac{1}{d_{b d}}} .
$$

This final expression is to be used in measurement precision estimations. Note that the weakest SNR component prevails in (32). An example of application is given in Fig. 11, with the same parameters as in Fig. 8.

Qualitatively speaking, two different SNR regimes are actually met here (since the baseline decorrelation effect appears, in this particular case, to remain negligible at all ranges).

- Near the vertical and at oblique angles, $d_{e x t}$ is very high. Hence, $d_{s l}$ predominates, and measurement fluctuations are then due to the shifting footprint effect.

- At low grazing angles, $d_{\text {ext }}$ decreases down to the maximum obtainable range. On the other hand, $d_{s l}$ is improving (with a strong maximum when pointing along its axis); the ambient noise influence is then prevalent.

\section{G. Near-Nadir Phenomena}

It is well known that phase-difference bathymetry suffers serious limitations near the direction orthogonal to the seafloor, resulting in a blind track underneath the sonar. Actually, the phasedifference measurements close to the specular is subject to specific problems, which are now analyzed. In the following, to retain easy notations and terminology, the normal to the seafloor will be denoted the "nadir" or "vertical" direction, with the implicit assumption that the seafloor is flat and horizontal.

Near vertical, the signal instantaneous footprint is at its largest extent in $x$, and so is the angle spreading effect; the corresponding depth error is then

$$
\delta z_{\text {vert }}=\frac{c T}{2 \sqrt{6}}
$$


Note that this last formula predicts a depth measurement error which has the order of magnitude of the equivalent signal length $c T / 2$.

The footprint shift effect described above is also at its maximum near nadir, as shown by (15), which gives for the equivalent SNR (neglecting the interferometer tilt)

$$
d_{s l} \rightarrow \frac{c T}{2 a}-1
$$

Note that this effect leads to a very important degradation when the interferometer spacing is of the order of magnitude of the equivalent signal length; possibly the footprints corresponding to the two receivers no longer have any common part! The effect upon depth measurement error is finally as follows for a square envelope:

$$
\delta z=\frac{H}{\pi \sqrt{\frac{c T}{2 a}-1}} \frac{\lambda}{a} .
$$

For a sine envelope, the corresponding results are

$$
\begin{aligned}
d_{s l} & \rightarrow \frac{1}{2}\left(\frac{c T}{\pi a}\right)^{2} \\
\delta z & =\frac{\sqrt{2} H \lambda}{c T} .
\end{aligned}
$$

With the parameters used in Section IV-C, this leads to a relative error for depth of around 5\% (square envelope) to $2.5 \%$ (sine envelope), which is clearly unacceptable according to the standard bathymetry requirements [17].

Practically, other effects intervene close to the vertical and make the reality even more intricate.

- The SNR considering additive noise is then at a very sharp maximum, due to the fact that both the instantaneous insonified area and the backscattering strength are at their highest; this clearly appears in Fig. 2. This may lead to problems in the receiver processing, where analog electronics and A/D converters may be unsuited for such high dynamics. Current systems use time gain control devices in order to avoid saturation bound to these signal peaks.

- Near vertical incidence, signal fluctuations are very strong, due to the large extent of the backscattering area which leads to a typical Rayleigh fluctuation regime; also, the BS variations with angle are then quite sharp.

- The directivity patterns of the arrays used in side-scan sonars are often designed to lower the received signal at vertical, partly to avoid cross-talk between the two sides of the sonar. Also, their response may be perturbed by their mechanical environment, since they are usually mounted on towed-fish flanks; the resulting masking effect may create phase perturbations.

For sonar systems using phase difference measurements, the poor performances associated with near-nadir bathymetry are due to the lack of across-track resolution at those incidences. This maximizes the angle spreading and shifting footprint effects, while it practically precludes the use of a sliding average on the time samples because of the low number of available samples. Neither can these systems resolve time-angle ambiguities met when various points of the seafloor topography are seen simultaneously at different angles, as it may be found in steep slope zones (canyons, ridges, cliffs). It seems that little can be done to improve this situation, considering the simple interferometers used in side-scan sonars; the best solution for a correct processing of these situations seems to take advantage of the angular scanning provided by a multibeam array processing.

\section{SUMmary}

An analysis of the physical causes of fluctuations in phase difference measurements leads to the conclusion that, in many practical cases, the shifting footprint effect may be a very noticeable contributor, concurrently with the baseline decorrelation; the relative importance of the two effects depends on the actual geometrical configuration. This is confirmed by numerical simulations showing the relevance of the shifting footprint concept and the validity of its analytical approximation.

The shifting footprint effect is bound to the echo physical structure. Hence, it seems that little can be done against it. Practically, however, things can be bettered, the basic principle being always to compensate on the receivers the time shift due to the target observation tilt angle. A variety of solutions, actual or potential, exists.

- Multibeam echosounders using a cylindrical array, for which the interferometer axis always coincides with the observed direction, do not suffer from the footprint shift effect.

- The other multibeam systems may get rid of the shift effect quite easily. The use of a $\mathrm{V}$-shaped reception array, e.g., tilted at $40^{\circ}$ to $50^{\circ}$ from vertical, should reduce the shift effect. Also, delays between time signals on the two subarrays can be compensated for during the beamforming operation, thus counteracting for the sliding footprint effect.

- For interferometric side-scan sonars, the problem is the most difficult to solve, since they cannot take advantage of beamforming for a first angle measurement. However, it seems feasible to artificially point the interferometer axis close to the instantaneous target direction by just delaying one of the two signals in order to correct the shift. This implies performing a first approximate estimation of bathymetry, then applying an artificial delay between the two receivers depending on the raw estimation of the instantaneous impact point angle, and, finally, computing the final phase differences with a minimized sliding footprint effect.

\section{APPENDIX I}

\section{ESTIMATION PRECISION ON A RAYLEIGH-FLUCTUATING SignAL}

Let us consider the general problem of a parameter estimation, using a stable signal corrupted with a Gaussian noise, $d$ being the SNR. The estimated parameter $\varepsilon$ is then a Gaussian random variable, whose variance is given by some version of the Cramer-Rao bound [8] under the form

$$
\operatorname{var}(\varepsilon)=\frac{K^{2}}{d}
$$


where $K$ depends on the type of measurement actually performed (for instance, in the case of a phase difference measurement, $K$ is simply equal to $\sqrt{2}$ ).

Now, if the signal is fluctuating according to a Rayleigh's distribution, it may be shown [9] that the estimation error is no longer Gaussian, but follows a Student's law whose density function is

$$
p(\varepsilon)=\frac{\sqrt{d}}{K}\left[2+\varepsilon^{2} \frac{d}{K^{2}}\right]^{-3 / 2} .
$$

The variance of this centered distribution is given by

$$
\operatorname{var}(\varepsilon)=\int_{-\infty}^{\infty} \frac{\sqrt{d}}{K}\left[2+\varepsilon^{2} \frac{d}{K^{2}}\right]^{-3 / 2} \varepsilon^{2} d \varepsilon
$$

which is readily integrated by parts and becomes

$$
\operatorname{var}(\varepsilon)=\frac{2 K^{2}}{d} .
$$

Hence, the standard deviation of the estimation error is increased by a factor $\sqrt{2}$ due to the Rayleigh's law fluctuation compared to a stable signal.

\section{APPENDIX II}

\section{GEOMETRICAl APPROACH OF ANGUlar SPREADING}

If the signal is emitted in the interval $[0, T]$ (the following holds for a wide-band system and $T$ is the signal duration after pulse compression), the active seafloor segment (along the $x$ axis) instantaneously insonified at a given time $t \geq(2 \mathrm{H} / \mathrm{c})+T$ depends on the equivalent signal length $c T / 2$ and angle $\theta$. Its length (see Fig. 5) is precisely given by

$$
\begin{aligned}
\Delta x & =\left|x_{t}-x_{t-T}\right| \\
& =\left| \pm\left[\left(\frac{H}{\cos \theta}+\frac{c T}{2}\right)^{2}-H^{2}\right]^{1 / 2}-H \tan \theta\right| \\
& \approx\left| \pm\left[H^{2} \tan ^{2} \theta+\frac{H c T}{\cos \theta}\right]^{1 / 2}-H \tan \theta\right|
\end{aligned}
$$

where the \pm depends on the sign of angle $\theta$, whose absolute value is related to time $t$ and altitude $H$ by

$$
\frac{c(t-T)}{2}=\frac{H}{\cos \theta} .
$$

In the approximate form of (A5), $c^{2} T^{2} / 4$ has been neglected with respect to terms featuring $H$. The $\Delta x$ maximal value is observed at the vertical $(\theta=0)$ and is then

$$
\Delta x_{\mathrm{vert}} \approx[H c T]^{1 / 2} .
$$

Actually, this value is doubled if no array directivity allows one to discriminate the two sides of the swath near nadir, since the pulse then covers both sides of the vertical. This diaphony risk exists for $\cos \theta \geq H /(H+c T / 2)$ (e.g., a limit angle of $7^{\circ}$ with the numerical application of Section III-C).
At sufficiently oblique incidences, a limited development of (A5) (considering $H^{2} \tan ^{2} \theta$ large compared to the other bracketed terms) leads to the well-known expression

$$
\Delta x_{\mathrm{obl}} \approx \frac{c T}{2|\sin \theta|}
$$

which is simply the projection of the equivalent signal length $c T / 2$ on the seafloor with respect to incident angle.

The angle spreading corresponding to the footprint size $\Delta x$ is easily obtained from the geometry of Fig. 5 as

$$
\Delta \theta=\frac{\Delta x}{H} \cos ^{2} \theta
$$

In oblique incidence, the previous expressions lead to

$$
\Delta \theta_{\mathrm{obl}}=\frac{c T}{2} \frac{\cos ^{2} \theta}{H|\sin \theta|}
$$

and, at the vertical, $(\theta \rightarrow 0)$

$$
\Delta \theta_{\text {vert }}=\arctan \left(\sqrt{\frac{c T}{H}}\right) \approx \sqrt{\frac{c T}{H}} .
$$

If the resultant signal is considered as coming from anywhere inside the $\Delta \theta$ angle sector, the observed angle will be a random variable, centered on the average angle $(\theta+(\Delta \theta / 2))$ and equally distributed over $\Delta \theta$; hence, its standard deviation is $\Delta \theta / \sqrt{12}$. Considering a supplementary degradation of $\sqrt{2}$ due to the Rayleigh fluctuating character of signals (see Appendix I), the final standard deviation in oblique incidence is

$$
\delta \theta=\frac{\Delta \theta}{\sqrt{6}}=\frac{c T}{2 \sqrt{6}} \frac{\cos ^{2} \theta}{H|\sin \theta|} .
$$

The corresponding error on depth $z$ is then given by

$$
\delta z=x \delta \theta=H \tan \theta \frac{c T}{2 \sqrt{6}} \frac{\cos ^{2} \theta}{H|\sin \theta|}=\frac{c T}{2 \sqrt{6}} \cos \theta
$$

which does not depend on the sonar altitude and is maximum near the vertical.

It is interesting, in (9) for the baseline decorrelation, to feature explicitly the $\Delta x$ footprint extent

$$
\eta=\frac{k a}{H} \frac{\Delta x}{2} \cos ^{2} \theta \cos (\theta-\psi) .
$$

Also, since $\eta$ is usually small, $d$ is approximately equal to

$$
d=\frac{6}{\eta^{2}} \text {. }
$$

Now, considering (7), giving the angle measurement error, and replacing $d$ by the above expressions, it becomes

$$
\delta \theta=\frac{\eta}{\pi \sqrt{6}} \frac{\lambda}{a} \frac{1}{\cos (\theta-\psi)}=\frac{c T}{2 \sqrt{6}} \frac{\cos ^{2} \theta}{H|\sin \theta|}
$$

which is exactly the geometrical expression given by (A12).

\section{ACKNOWLEDGMENT}

The author wishes to thank C. Sintes (GESMA, Brest, France) for discussions about the work presented here, and for providing 
the idea of the optical analogy. The associate editor and the reviewers are also acknowledged for suggesting various improvements of the paper, particularly for proposing the topic of Section IV-D.

\section{REFERENCES}

[1] C. de Moustier, "State of the art in swath bathymetry survey systems," Int. Hyd. Rev., vol. 65, pp. 25-54, 1988.

[2] _ _ "Signal processing for swath bathymetry and concurrent seafloor acoustic imaging," in Acoustic Signal Processing for Ocean Exploration, J. M. F. Moura and I. M. G. Lourtie, Eds., 1993, pp. 329-354.

[3] Ph. Denbigh, "Swath bathymetry: Principles of operation and an analysis of errors," IEEE J. Oceanic Eng., vol. 14, pp. 289-298, Oct. 1989.

[4] J. G. Blackinton, "Bathymetric mapping with SeaMARC II: An elevation-angle measuring side-scan sonar system," Ph.D. thesis, Univ. of Hawaii, 1986.

[5] Ph. Denbigh, "Signal processing strategies for a bathymetric sidescan sonar," IEEE J. Oceanic Eng., vol. 19, pp. 382-390, July 1994.

[6] J. G. Blackinton, "Bathymetric resolution, precision and accuracy considerations for swath bathymetry mapping sonar systems," in IEEE Oceans '91, 1991, pp. 550-557.

[7] "Simrad EM 1000-Hydrographic Echosounder-Product Description," Simrad Subsea A/S, Horten, Norway, Simrad Document PZ2415E, 1992.

[8] W. S. Burdic, Underwater Sound System Analysis. Englewood Cliffs, NJ: Prentice-Hall, 1984, ch. 11.7, 13.3.3.

[9] M. Carpentier, Radar-Bases Modernes. Paris, France: Masson, 1984, ch. 6.5.2.2.

[10] S. Dugelay, X. Lurton, and J. M. Augustin, "A new method for seafloor characterization with multibeam echosounders: Image segmentation using angular backscattering," in 3rd Eur. Conf. Underwater Acoustics, 1996, pp. 439-444.

[11] L. Hellequin, "Statistical characterization of multibeam echo sounder data," in Proc. IEEE Oceans'98, 1998.
[12] M. Masnadi-Shirazi et al., "Differential phase estimation with the SeaMARC II bathymetric sidescan sonar system," IEEE J. Oceanic Eng. vol. 17, pp. 239-251, July 1992.

[13] P. Cervenka and C. de Moustier, "Postprocessing and corrections of bathymetry derived from sidescan sonar systems: Application with SeaMARC II," IEEE J. Oceanic Eng., vol. 19, pp. 619-629, Oct. 1994.

[14] G. Jin and D. Tang, "Uncertainties of differential phase estimation associated with interferometric sonars," IEEE J. Oceanic Eng., vol. 21, pp. 53-63, Jan. 1996.

[15] C. Sintes, "Bruit interférométrique pour sonar latéral," Groupe d'Etudes Sous-Marines de l'Atlantique, Brest, Tech. Rep. GESMA RT 4336, 1999.

[16] V. V. Ol'chevskii, Characteristics of Sea Reverberation. New York, NY: Consultant Bureau, 1967.

[17] "IHO standards for Hydrographic surveys-4th edition," in Special Publication no. 44, Monaco: International Hydrographic Bureau, 1998.

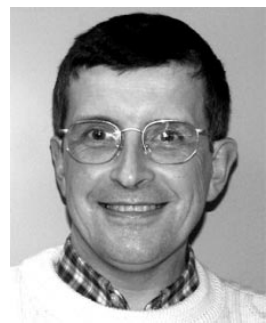

Xavier Lurton was born in Bordeaux, France, in 1955. He received the Ph.D. degree in applied acoustics from the Université du Maine, Le Mans, France, in 1979.

He worked from 1981 to 1989 with Thomson-Sintra ASM, mainly specializing in the field of underwater sound propagation modeling for naval applications. In 1989, he joined IFREMER, the French oceanology research agency. After working on various acoustical oceanography applications (ocean tomography, telemetry, fisheries sonar) and managing the IFREMER acoustics laboratory for five years, he is now in charge of a research program on seafloor characterization using multibeam echosounders, his current interests being both in the physics of seabed backscattering and in sonar signal processing. 